\title{
Video-otoscopy recordings for diagnosis of childhood ear disease using telehealth at primary health care level
}

\author{
Biagio $\mathrm{L}^{1}$, Swanepoel de $\mathrm{W}^{2}$, Laurent $\mathrm{C}^{3}$, Lundberg $\mathrm{T}^{4}$ \\ ${ }^{1}$ Department of Communication Pathology, University of Pretoria, South Africa leigh.biagio@up.ac.za. \\ ${ }^{2}$ Department of Communication Pathology, University of Pretoria, South Africa Ear Sciences Centre, \\ School of Surgery, University of Western Australia, Nedlands, Australia Ear Science Institute Australia, \\ Subiaco, Australia. \\ ${ }^{3}$ Department of Communication Pathology, University of Pretoria, South Africa ENT Unit, Department of \\ Clinical Science, Umeå University, Sweden. \\ ${ }^{4}$ Family Medicine, Department of Public Health and Clinical Medicine, Umeå University, Sweden.
}

\section{Summary}

We studied the diagnosis made by an otologist and general practitioner [GP]) from video-otoscopy recordings on children made by a telehealth facilitator. The gold standard was otomicroscopy by an experienced otologist. A total of 140 children (mean age 6.4 years; $44 \%$ female) were recruited from a primary health care clinic.

Otomicroscopic examination was performed by an otologist. Video-otoscopy recordings were assigned random numbers and stored on a server. Four and eight weeks later, an otologist and a GP independently graded and made a diagnosis from each video recording. The otologist rated the quality of the video-otoscopy recordings as acceptable or better in $87 \%$ of cases. A diagnosis could not be made from the video-otoscopy recordings in $18 \%$ of ears in which successful onsite otomicroscopy was conducted. 
There was substantial agreement between diagnoses made from video-otoscopy recordings and those from onsite otomicroscopy (first review: otologist $\kappa=0.70$ and GP $\kappa=0.68$; second review: otologist $\kappa=0.74$ and GP $\kappa=0.75)$. There was also substantial inter-rater agreement $(\kappa=0.74$ and 0.74 at the two reviews) and intra-rater agreement $(\kappa=0.77$ and 0.74 for otologist and GP, respectively).

A telehealth facilitator, with limited training, can acquire sufficient quality videootoscopy recordings in children for asynchronous diagnosis. Remote diagnosis was similar to face-to-face diagnosis in inter- and intra-rater variability.

\section{Keywords}

Video-otoscopy, telehealth, telemedicine, ear disease, primary health care, facilitator, sub-Saharan Africa, paediatric

\section{Introduction}

Telehealth has been proposed as a means of bringing ear and hearing health services to people in underserved regions. ${ }^{1,2}$ The nature of the otitis media burden, as the most common ear disease, differs greatly between developed and developing countries. ${ }^{3,4}$ 
Acute otitis media (AOM) incidence in Sub-Saharan African, South Asia and Oceania is two to eight times higher than in other world regions. ${ }^{5}$ The prevalence of chronic suppurative otitis media (CSOM) in Africa was classified as high by the World Health Organisation (WHO), ${ }^{6}$ with sub-Saharan Africa presenting with the second highest global incidence of CSOM. ${ }^{3}$ Unfortunately, estimates of otolaryngologists available to serve populations in sub-Saharan Africa vary from 1 to every 250,000 to 7.1 million people. ${ }^{4,7}$ Early diagnosis and subsequent treatment of ear disease is therefore particularly difficult to achieve, because hearing health services and hearing professionals are very limited. ${ }^{8}$

Specialist services may potentially be extended to rural and underserved areas by use of video-otoscopy used within a telehealth framework. ${ }^{2}$ By incorporating video-otoscopy in telehealth clinics, digitized images or recordings can be stored and forwarded to otolaryngologists anywhere in the world for asynchronous assessment. ${ }^{2,9,10}$ In the absence of otolaryngologists, general practitioners (GPs), as first line physicians, may be required to make diagnoses from video-otoscopy recordings. ${ }^{9}$ Use of video-otoscopy within telehealth programs has been reported using video-otoscopy images and recordings, and through both synchronous and asynchronous methods of evaluation. ${ }^{10-17}$ Previous studies have demonstrated that asynchronous video-otoscopy images are 
equivalent in quality, and offer average to good diagnostic concordance, with onsite otoscopy. ${ }^{2,9,11,12,15-18}$

In a recent study, video-otoscopy images in adult patients were acquired by an ear and hearing telehealth facilitator with no formal health care education. ${ }^{10}$ Asynchronous diagnosis from video-otoscopy images yielded moderate concordance with onsite diagnosis made by the same otolaryngologist. A limitation of video-otoscopy images was identified as the lack of depth perception, which may well be addressed by making video-otoscopy recordings of a few seconds in length. ${ }^{10,11}$

Both synchronous and asynchronous video-otoscopy recordings were employed by Smith et al. ${ }^{14,17}$ Smith et al. ${ }^{14,17}$ reported a higher diagnostic concordance with synchronous, compared to asynchronous diagnosis using video-otoscopy recordings, with the advantage of instantaneous feedback from the remote specialist. Synchronous diagnosis is not always possible however, due to time constraints of specialists, and even time differences between locations. Synchronous diagnosis requires high speed broadband connectivity which is also unavailable in areas such as sub-Saharan Africa. Video-otoscopy recordings in the study by Smith et al. ${ }^{14,17}$ were acquired by a paediatrician or a research nurse, neither of which is likely be available in remote health clinics. 
The capture of video-otoscopy information at primary health care level may be completed by a telehealth facilitator with limited or no formal health training. ${ }^{10}$ Utilising telehealth facilitators to acquire video-otoscopy for remote asynchronous interpretation may be a powerful tool to identify pathology early, and make appropriate recommendations whilst avoiding excessive waiting times and costs related to travelling. ${ }^{10,19}$ This is particularly important for children in remote areas who may be more prone to ear disorders such as otitis media. ${ }^{5,20}$

In studies comparing asynchronous diagnosis using video-otoscopy, conventional otoscopy has usually served as the gold standard reference. ${ }^{10,14}$ The most ideal method under which an ear examination may be performed is considered to be through use of an operating otomicroscope with an ear canal free of cerumen. ${ }^{21}$ In clinical practice this is rarely available to a clinicians. ${ }^{12}$ Nevertheless, the selection of otomicroscopy instead of conventional otoscopy is expected to provide a more accurate gold standard for comparison of diagnostic concordance with video-otoscopy recordings.

The aim of this study was therefore to investigate otologist and general practitioner (GP) interpretations of asynchronous video-otoscopy recordings made by an ear and hearing telehealth clinic facilitator, compared to onsite otomicroscopy, in a paediatric population. Video-otoscopy interpretations by a GP were included due to the limited 
number of otolaryngologists available in sub-Saharan Africa leaving diagnosis to GP's in many cases. ${ }^{4,7}$

\section{Materials and Methods}

The study followed a within-subject comparative design and was conducted following approval from the institutional ethics committee at the University of Pretoria. A convenience sample of 140 children aged two to 16 years of age (range 2 to 15.8 years; mean age $6.4 \pm 3.5$ years; $44.3 \%$ female) were recruited from a primary health care clinic. Witkoppen Health and Welfare Centre is a primary health care clinic that provides services to the Diepsloot community north of Johannesburg, South Africa.

Diepsloot is a densely populated, poor socio-economic settlement with estimates suggesting that more than $90 \%$ of the population is unemployed. ${ }^{22}$ There is no hospital in Diepsloot despite a very high prevalence rate of HIV and associated TB infections. ${ }^{22}$

Participants were recruited from the entire paediatric population attending the clinic irrespective of reason for attendance. Caregivers were informed of the procedure and were required to give consent before any data collection commenced. Biographical 
information and history of ear ache, ear discharge or hearing loss during the two weeks prior to participation in the study was then recorded.

\section{Otomicroscopic examination}

Otomicroscopy was completed of participants' ears by an experienced otologist ( $>35$ years of practice). Onsite otomicroscopy examination was considered the gold standard diagnosis. Cerumen was removed manually in order to obtain a clear view of the tympanic membrane. Cerumen removal was discontinued if any discomfort was noted. Thereafter, observations regarding ear canal obstruction, presence of any discharge, tympanic membrane patency, translucency and position, as well as the concluding diagnosis were documented.

Diagnosis of tympanic membrane status was based on visual otomicroscopic examination alone, without objective assessment of the tympanic membrane mobility. The types of otitis media were classified as either AOM, otitis media with effusion (OME) or CSOM. Classification of the types of otitis media were recorded according to the following criteria: AOM diagnosis was based on clinical data (e.g. rapid onset of fever, otalgia, or irritability) and otomicroscopic findings of either a bulging intact tympanic membrane, or a wet, contourless, perforated tympanic membrane. ${ }^{23}$ Diagnosis of OME was based on suspicion of sero-mucoid or serous effusion in the middle ear 
(completely filled or air-fluid level or bubbles), with an intact tympanic membrane without symptoms of acute infection. ${ }^{23} \mathrm{CSOM}$ diagnosis was made based on the presence of perforation, deep not fully visible retraction pocket or cholesteatoma) with or without purulent discharge. ${ }^{5}$ Ears without disease were classified as 'normal'.

\section{Video-otoscopy recordings}

Following otomicroscopy, video-otoscopy recordings of nine to 33 seconds in length each (mean $25.6 \mathrm{~s}$ ) were completed by an ear and hearing telehealth clinic facilitator from each ear of participants. The facilitator had no formal health care or tertiary education. Prior to data collection onsite training was provided over a two day period by the otologist on how to conduct video-otoscopy recordings. Training included participant positioning, visual inspection of external ear, appropriate hand position, manipulation of direction of speculum, focus adjustment, recording capture, videootoscope software use, and equipment cleansing.

Data collection, which included acquisition of case histories, onsite otomicroscopy result and acquisition of video-otoscopy recordings by the facilitator (see Figure 1), was completed over a period of two weeks. 


\section{Equipment}

Otomicroscopy was done using a Leica M525 F40 surgical otomicroscope with a 6:1 zoom magnification (1.2 to $12.8 x$ ) and 300-watt xenon fibre optic illumination. An AMH-EUT Dino-Lite Pro Earscope (USB), with a 3, 4 or 5mm speculum was used to acquire the video-otoscopy recordings (Figure 1). The Dino-Lite Pro made use of a

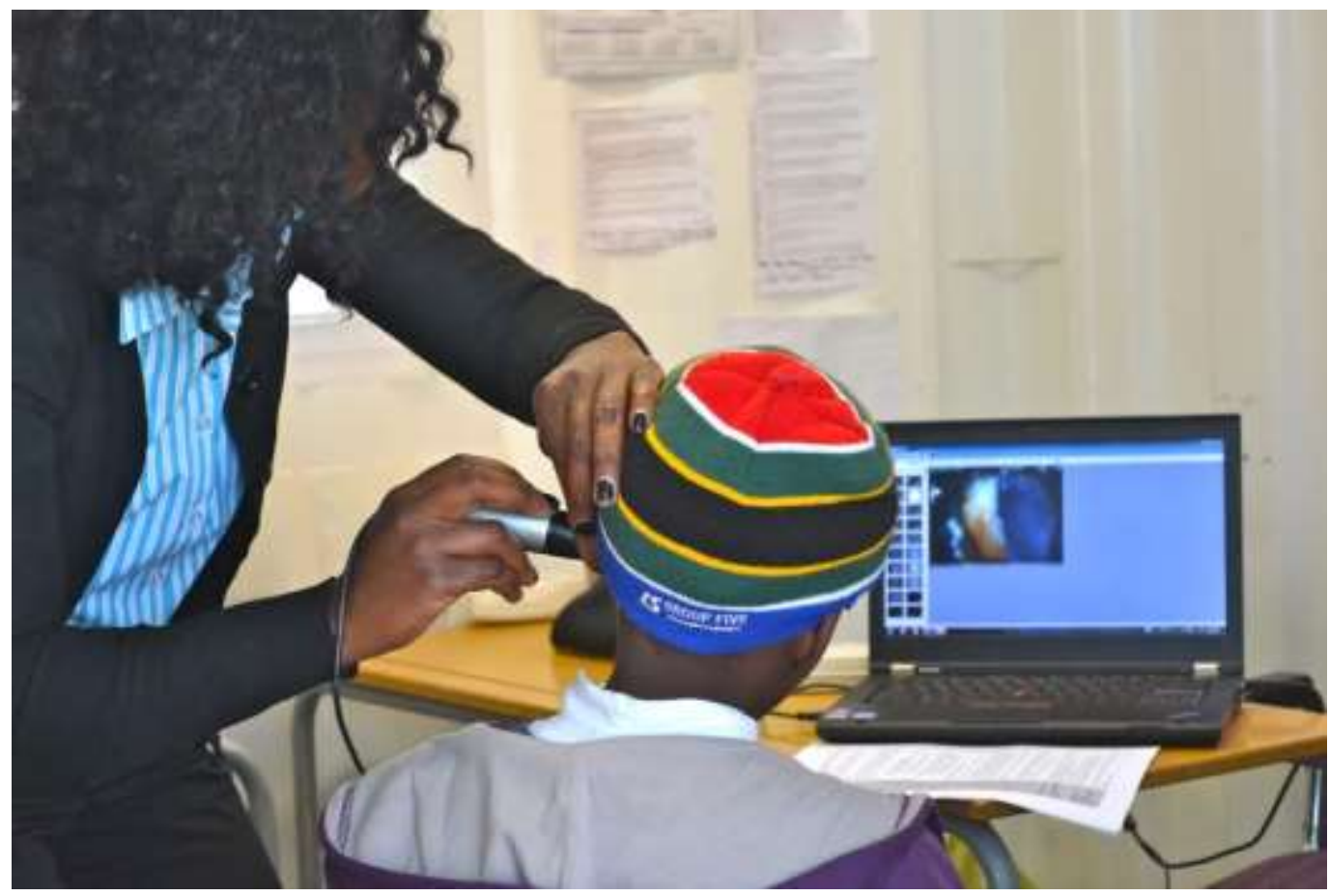

Figure 1. Hearing telehealth clinic facilitator completing video-otoscopy recording for a child.

LED light, a magnification rate of 10 to $20 \mathrm{x}$, a frame rate 30 frames per second, and 1.3 megapixel resolution. The Dino-Lite Pro video-otoscope was attached, via a USB video cable, to a Lenovo ThinkPad 2.0 running Windows 7 via USB 2.0 interface.

DinoCapture 2.0 software (AnMo Electronics Corporation) version 1.2.7 was used to 
record and view the video-otoscopy recordings. The recordings were saved as WMV files and ranged from 0.85 to $7.61 \mathrm{MB}$ in size (mean = 3.6 MB).

\section{Asynchronous assessment}

After data collection was completed, video-otoscopy recordings, the participant's demographic information and case history based of ear ache, discharge and perceived hearing loss were uploaded to a secure server, using a web-based file hosting service (Dropbox). Recordings were assigned random numbers by an independent investigator prior to the first asynchronous evaluation (review one) and again prior to the second asynchronous evaluation (review two). Four and eight weeks after onsite data collection, an otologist (the same otologist who performed onsite otomicroscopy) and a GP, who were blinded to the randomised numbering of recordings, accessed the server. Each rater was required to independently grade the video-otoscopy recordings, to make observations regarding ear canal obstruction, presence of secretion, tympanic membrane patency, translucency and position, and diagnosis from each video recording. The overall image quality was graded (0 to 2 ) with reference to image focus, light, cerumen

and composition. ${ }^{9}$ A grading of 0 indicated that the image quality was not acceptable, and it was not possible to assess the entire tympanic membrane and to set a diagnosis. An image graded 1 indicated an acceptable image quality, enabling evaluation of the status of the tympanic membrane. An excellent video-otoscopy image was graded 2, 
indicating high image quality, with tympanic membrane easily assessable. The asynchronous diagnosis was made using the same classification as was used during onsite otomicroscopy.

The asynchronous assessments were logged on an electronic spread sheet and uploaded to the server once completed. The delay in asynchronous assessment was included to counter the possibility of a memory effect for images and onsite diagnoses made. The second asynchronous assessment four weeks after the first allowed for assessment of intra-rater correspondence.

\section{Analyses}

Descriptive statistics were used to describe the mean recording quality rating for videootoscopy recordings and the frequency with which ear canal obstruction and presence of secretion was identified, as well as the tympanic membrane patency, translucency and position. ${ }^{24}$ The frequency of diagnosis of the different types of otitis media was also measured. For analysis of ears where an asynchronous diagnosis could not be made, labelled as not possible to diagnose (NPD), participants were divided into a younger and an older age group. The two groups represented preschool children ( 2 to 5 years of age) and children in formal education (6 to 16 years of age). Comparisons between the 
number of undiagnosed ears and the age group were made using Pearson's chi-squared test with a probability of $5 \%$ considered to be significant. Pearson's chi-squared test was also used for comparing the quality grading of video-otoscopy recordings acquired during the first and during the second week of data collection.

Kappa statistic ( $\kappa)$ was used to quantify concordance of diagnosis made from onsite otomicroscopic examination and asynchronous video-otoscopy recordings, inter- and intra-rater concordance of asynchronous and diagnosis from video-otoscopy recordings. For diagnostic concordance between onsite examination and asynchronous videootoscopy recordings, calculations of sensitivity, specificity, positive and negative predictive values ( $\mathrm{n}=176$ ears), all ears where a diagnosis could not be made from either assessment method were excluded from the kappa calculations. For inter- and intra-rater concordance ( $n=249$ ears), the ears where a diagnosis could not be made during asynchronous assessment were excluded from the kappa calculations. The concordance was based upon the range in which kappa statistic matches: "poor agreement" $(\kappa<0.00)$, "slight agreement" $(\kappa=0.01-0.20)$, "fair agreement" $(\kappa=0.21-$ $0.40)$, “moderate agreement" ( $\kappa=0.41-0.60)$, “substantial agreement" $(\kappa=0.61-0.80)$, "almost-perfect agreement" $(\kappa=0.81-1.00){ }^{25}$ 
By classifying the diagnosis as normal or abnormal, the sensitivity, specificity, positive and negative predictive value of asynchronous diagnosis from video-otoscopy recordings acquired by the facilitator was calculated with reference to otomicroscopic examination by the otologist as the 'gold standard'.

\section{Results}

Four participants did not co-operate for onsite otomicroscopy. Otomicroscopy was therefore completed for 136 participants (272 ears). One of the 136 participants did not co-operate for video-otoscopy in either ear, while another participant did not allow video-otoscopy to be completed in one ear. Therefore video-otoscopy recordings were carried out on 135 participants, and 269 ears.

During asynchronous assessment, the otologist graded video-otoscopy recordings as unacceptable in $13 \%$ of ears for the first and second review whilst the GP graded $22.3 \%$ and $26.4 \%$ unacceptable for the two review sessions, respectively (Table 1). To examine whether experience improved the quality of recordings by the facilitator, videootoscopy gradings were assessed separately for recordings acquired during the first and second week of data collection (Table 1). Both the otologist and the GP judged more video-otoscopy recordings as excellent in quality in the second compared to the first week of data collection (otologist mean number of excellent ratings for each review 
Table 1. Asynchronous grading of video-otoscopy recordings acquired during the first and second week of data collection. (R1 - Review 1; R2 - Review 2)

\begin{tabular}{|c|c|c|c|c|c|c|c|c|c|c|c|c|c|}
\hline & \multirow{3}{*}{$\begin{array}{c}\text { Recording } \\
\text { grading }\end{array}$} & \multicolumn{4}{|c|}{$\begin{array}{c}\text { Week } 1(\%) \\
(n=134 \text { ears })\end{array}$} & \multicolumn{4}{|c|}{$\begin{array}{c}\text { Week } 2(\%) \\
(n=135 \text { ears })\end{array}$} & \multicolumn{4}{|c|}{$\begin{array}{c}\text { Total }(\%) \\
(n=269 \text { ears })\end{array}$} \\
\hline & & \multicolumn{2}{|c|}{ Otologist } & \multicolumn{2}{|c|}{ GP } & \multicolumn{2}{|c|}{ Otologist } & \multicolumn{2}{|c|}{ GP } & \multicolumn{2}{|c|}{ Otologist } & \multicolumn{2}{|c|}{ GP } \\
\hline & & R1 & $\mathbf{R 2}$ & R1 & $\mathbf{R 2}$ & R1 & $\mathbf{R} 2$ & R1 & $\mathbf{R} 2$ & R1 & $\mathbf{R} 2$ & R1 & $\mathbf{R} 2$ \\
\hline \multirow{3}{*}{$\begin{array}{l}\mathbf{0} \\
1 \\
2\end{array}$} & \multirow{3}{*}{$\begin{array}{l}\text { Unacceptable } \\
\text { Acceptable } \\
\text { Excellent }\end{array}$} & 14.2 & 13.4 & 22.4 & 26.1 & 11.9 & 12.6 & 22.2 & 26.7 & 13.0 & 13.0 & 22.3 & 26.4 \\
\hline & & 79.1 & 84.3 & 51.5 & 49.3 & 77.0 & 79.3 & 47.4 & 40.7 & 78.1 & 81.8 & 49.4 & 45.0 \\
\hline & & 6.7 & 2.3 & 26.1 & 24.6 & 11.1 & 8.1 & 30.4 & 32.6 & 8.9 & 5.2 & 28.3 & 28.6 \\
\hline
\end{tabular}

$4.5 \%$ and $9.6 \%$ for week one and two respectively; GP mean number of excellent ratings $25.4 \%$ and $31.5 \%$ respectively). This was true at both the first and second review sessions. The improvement in quality ratings between the first and second week of data collection was not statistically significant however ( $p>0.05$; Chi-squared test).

Mean intra-rater agreement at review one and two, was $87.0 \%$ and $73.6 \%$ on recordings labelled as either acceptable or excellent for the otologist and GP respectively. Interrater agreement on video-otoscopy recordings graded as either acceptable or excellent was $76.6 \%$ for review one, and $72.1 \%$ for review two. Rater disagreement was recorded regarding poor quality video-otoscopy recordings in $9.3 \%$ to $13.4 \%$ of ears for review one and two respectively. 
Poor video-otoscopy recordings were more common in younger children $(2-5$ years of age), compared to older children (6 -15 years of age) for both raters (Figure 2), but was not statistically significant ( $\mathrm{p}>0.05$; Chi-squared test).

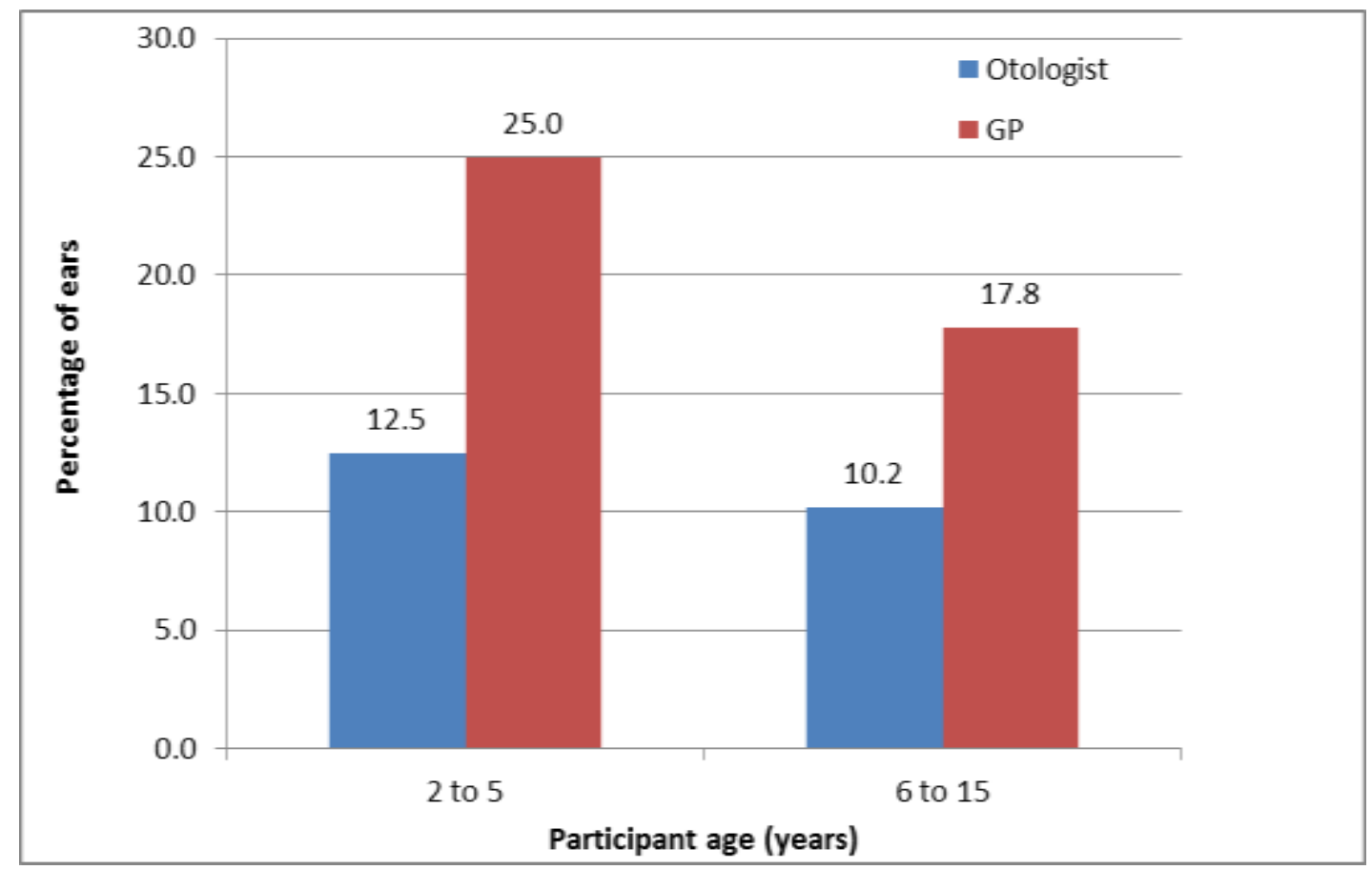

Figure 2. Video-otoscopy recordings rated poor quality as a function of participant age. $(\mathrm{n}=269$ ears $)$

During onsite assessment, manual cerumen removal was deemed necessary and attempted for $36.0 \%$ of participants $(23.5 \%$ of ears) in order to obtain a clear view of the tympanic membrane for otomicroscopic diagnosis. After reasonable attempts were made to remove any cerumen without causing discomfort, cerumen still partially or completely occluded the ear canal in $12.9 \%$ of participants for either or both ears $(7.7 \%$ 
Table 2. Onsite diagnoses made by the otologist using otomicroscopy compared to asynchronous diagnoses made by the otologist and GP using video-otoscopy recordings. (R1 - Review 1; R2 - Review 2)

\begin{tabular}{|c|c|cc|cc|}
\hline & \multicolumn{2}{|c|}{$\begin{array}{c}\text { Onsite diagnosis } \\
\mathbf{n = 1 3 6}(\mathbf{2 7 2} \text { ears) }\end{array}$} & \multicolumn{3}{|c|}{$\begin{array}{c}\text { Asynchronous diagnosis } \\
\mathbf{n}=\mathbf{1 3 5}(\mathbf{2 6 9} \text { ears) }\end{array}$} \\
\cline { 2 - 6 } & Otologist (\%) & \multicolumn{2}{|c|}{ Otologist (\%) } & \multicolumn{2}{c|}{ GP(\%) } \\
& & $\mathbf{R 1}$ & $\mathbf{R 2}$ & $\mathbf{R 1}$ & $\mathbf{R 2}$ \\
\hline Normal & $65.5(75.8)$ & $63.0(58.4)$ & $68.1(62.1)$ & $65.9(58.0)$ & $69.6(60.6)$ \\
Otitis media: & $21.6(16.5)$ & $25.2(16.7)$ & $20.8(14.5)$ & $20.0(14.5)$ & $17.1(12.6)$ \\
AOM & $1.4(0.7)$ & $0.0(0.0)$ & $1.5(0.7)$ & $0.7(0.4)$ & $1.5(0.7)$ \\
CSOM & $5.8(4.8)$ & $9.6(6.7)$ & $8.9(6.3)$ & $7.4(4.8)$ & $5.2(4.1)$ \\
OME & $14.4(11.0)$ & $15.6(10.0)$ & $10.4(7.5)$ & $11.9(9.3)$ & $10.4(7.8)$ \\
NPD & $12.9(7.7)$ & $11.8(24.9)$ & $11.1(23.4)$ & $14.1(27.5)$ & $13.3(26.8)$ \\
\hline
\end{tabular}

AOM = acute otitis media; $C S O M=$ chronic suppurative otitis media; OME = otitis media with effusion; $N P D=$ not possible to diagnose

of ears) preventing a diagnosis from being made (Table 2). During asynchronous diagnosis, the inability of the otologist and GP to make a diagnosis was due to partial or complete occlusion of the ear canal (due to lack of visualisation of the entire tympanic membrane), or poor video-otoscopy recording quality. At review one and two respectively, the otologist was unable to make a diagnosis in $24.9 \%$ and $23.4 \%$ of ears. The GP was unable to make a diagnosis for $27.5 \%$ and $26.8 \%$ ears at asynchronous review one and two, respectively. Asynchronous diagnosis from video-otoscopy recordings could therefore not be made from a mean (calculated from mean between reviews of both raters) $18 \%$ of ears for whom successful onsite otomicroscopy was conducted. 
Otitis media was identified in $12.6 \%$ to $16.7 \%$ of ears, with OME being the most common type of otitis media (7.5\% to $10.0 \%$ of ears), followed by CSOM ( $4.1 \%$ to $6.7 \%$ of ears). The otologist reported a larger number of ears with CSOM during asynchronous assessment (mean number of ears with CSOM diagnosed at review one and two $6.5 \%$ ) compared to either onsite otomicroscopy (CSOM of $4.8 \%$ of ears) or asynchronous video-otoscopy assessment by the GP (mean CSOM ears 4.5\%).

Table 3. Concordance of asynchronous diagnosis using video-otoscopy recordings by the otologist and GP compared to onsite otomicroscopy. ( $n=176$ ears; R1 - Review 1; R2 - Review 2)

\begin{tabular}{|l|l|c|c|}
\hline \multicolumn{2}{|c|}{} & $\begin{array}{c}\text { Concordance between asynchronous video- } \\
\text { otoscopy recordings }\end{array}$ \\
\cline { 3 - 4 } \multicolumn{2}{|c|}{} & Kappa value & Asymp. std. error \\
\hline R1 & Otologist & 0.702 & 0.070 \\
& GP & 0.679 & 0.074 \\
\hline R2 & Otologist & 0.740 & 0.068 \\
& GP & 0.745 & 0.069 \\
\hline
\end{tabular}

Asymp. std. error $=$ Asymptotic standard error

There was substantial agreement between asynchronous video-otoscopy diagnoses compared to onsite otomicroscopic diagnoses (Table 3). A slightly higher diagnostic concordance was found at review two for both the otologist $(\kappa=0.740)$ and for the GP $(\kappa=0.745)$ than for review one (otologist $\kappa=0.702 ;$ GP $\kappa=0.679)$.

Agreement between diagnosis made using asynchronous video-otoscopy recordings was substantial between raters $(\kappa=0.737$ and 0.735 at review one and two) and within raters $(\kappa=0.773$ and 0.737 for otologist and GP respectively; Table 4). 
Table 4. Concordance of asynchronous diagnosis using video-otoscopy recordings between and within the otologist and GP. ( $\mathrm{n}=249$ ears; R1 - Review 1; R2 - Review 2)

\begin{tabular}{|l|l|c|c|}
\hline \multicolumn{2}{|c|}{} & \multicolumn{2}{c|}{$\begin{array}{r}\text { Concordance between asynchronous video- } \\
\text { otoscopy recordings }\end{array}$} \\
\cline { 3 - 4 } \multicolumn{2}{|l|}{} & Kappa value & Asymp. std. error \\
\hline $\begin{array}{l}\text { Inter-rater } \\
\text { diagnosis }\end{array}$ & R1 & 0.737 & 0.040 \\
& R2 & 0.735 & 0.040 \\
\hline $\begin{array}{l}\text { Intra-rater } \\
\text { diagnosis }\end{array}$ & Otologist & 0.773 & 0.038 \\
& GP & 0.737 & 0.040 \\
\hline
\end{tabular}

Asymp. std. error = Asymptotic standard error

Specificity and negative predictive values were higher than sensitivity and positive predictive values for asynchronous video-otoscopy interpretations for both raters (Table 5). The sensitivity of the asynchronous interpretation of the video-otoscopy recordings was slightly better for the otologist (mean sensitivity of 77.6\%) than for the GP (mean sensitivity of $72.4 \%$ ). Slightly higher positive predictive values were determined from

Table 5. Sensitivity, specificity, positive and negative predictive values (\%) for normal and abnormal classifications of asynchronous video-otoscopy recordings as assessed by an otologist and GP. ( $\mathrm{n}=176$ ears; R1 - Review 1; R2 - Review 2)

\begin{tabular}{|l|l|c|c|c|c|}
\hline \multicolumn{2}{|c}{} & Sensitivity & Specificity & $\begin{array}{c}\text { Positive } \\
\text { predictive value }\end{array}$ & $\begin{array}{c}\text { Negative } \\
\text { predictive value }\end{array}$ \\
\hline Otologist & R1 & 79.3 & 93.2 & 69.7 & 95.8 \\
& R2 & 75.9 & 95.9 & 78.6 & 95.3 \\
& Mean & 77.6 & 94.6 & 73.8 & 95.5 \\
\hline GP & R1 & 72.4 & 95.2 & 75.0 & 94.6 \\
& R2 & 72.4 & 98.0 & 87.5 & 94.7 \\
& Mean & 72.4 & 96.6 & 80.8 & 94.7 \\
\hline
\end{tabular}


the GP evaluation of asynchronous video-otoscopy recordings (positive predictive value of $80.8 \%$ ) compared to those of the otologist (positive predictive value of $73.8 \%$ ).

\section{Discussion}

Quality of video-otoscopy recordings

Video-otoscopy recordings acquired by the telehealth facilitator were rated as acceptable or better in quality for $73.6 \%$ to $87.0 \%$ of cases by the otologist and GP. This is comparable to the proportion of adequate video-otoscopy cases acquired by a nurse and an otolaryngologist in previous studies (viz. $75 \%$ to $85 \%$ recordings judged to be adequate in quality). ${ }^{10,14}$ The similarity in quality judgements reported by the current study and in previous studies are of consequence for three reasons. Firstly, the telehealth facilitator who captured the video-otoscopy recordings had no formal health care training unlike the health care personnel used in earlier studies. ${ }^{10,14}$ Secondly, videootoscopy recordings in the current study were found to be equivalent in quality compared to the video-otoscopy images acquired previous. ${ }^{10}$ Thirdly, the present study targeted a paediatric population who were likely to be less co-operative than the adult population of Biagio et al. ${ }^{10}$ 
The number of video-otoscopy recordings rated excellent by the otologist and GP was higher (albeit not significantly) for recordings acquired during the second week and may suggest a learning effect with increased experience by the facilitator (mean excellent gradings by otologist in week one increased from $4.5 \%$ to $9.6 \%$; mean excellent gradings by GP increased from $25.4 \%$ to $31.5 \%$ ). An improvement in quality of videootoscopy images acquired over time was also demonstrated by Lundberg et al. ${ }^{9}$ The quality of video-otoscopy recordings is likely to be impacted by the amount of training and level of proficiency of the person acquiring the recordings, which, ultimately, affects the diagnostic accuracy of video-otoscopy recordings as a means of asynchronous diagnosis of ear disease.

Asynchronous diagnosis from video-otoscopy recordings could not be made from $18 \%$ of ears for whom successful onsite otomicroscopy was completed in the current study. This may be attributable to several factors including poor video quality, insufficient visualisation of the entire tympanic membrane, and / or partial occlusion of the ear canal by cerumen. In previous studies, when the entire tympanic membrane could not be visualised, Eikelboom et al. ${ }^{15}$ and Biagio et al. ${ }^{10}$ reported the presence of partial or total cerumen occlusion rather than stating that a diagnosis could not be made. Kokesh et al. ${ }^{11}$ remarked that images were discarded due to poor image quality or cerumen but did not indicate how many. Use of cerumen removal strategies is likely to reduce the 
number of undiagnosed ears from asynchronous video-otoscopy if they are performed prior to the recording. Providing a cerumenolytic a few days earlier may facilitate both manual removal of the cerumen, and removal using syringing. Furthermore cerumen removal may be performed by a nurse at a clinic immediately prior to video-otoscopy recording.

Poor video-otoscopy image quality or a lack of sufficient information was reported as the reason for being unable to diagnose $14 \%$ of ears in the study by Patricoski and colleagues compared to $18 \%$ in the current study. ${ }^{12}$ In a previous study, Biagio et al. ${ }^{10}$ reported a lower number of ears where asynchronous diagnosis could not be made from video-otoscopy images acquired by a telehealth facilitator in adult subjects (10\% of images). This difference may in part be attributable to participant age (paediatric versus adult participants) and consequent variability in co-operation.

\section{Diagnostic accuracy using video-otoscopy recordings}

Agreement between onsite otomicroscopy and asynchronous evaluation of videootoscopy recordings in the present study was substantial $(\kappa=0.679-0.745)$ and equivalent to concordance previously reported using video-otoscopy images and otoscopy $(\kappa=0.64-0.76) .{ }^{11,12}$ The correspondence of concordance scores suggest that the telehealth facilitator in the present study, with no formal health care training, was 
capable of acquiring video-otoscopy recordings comparable to personnel with formal health care education who completed video-otoscopy in previous reports. ${ }^{11,12}$

Validity of asynchronous diagnoses in this study is supported by similar correspondence with onsite otoscopy reported by Smith and colleagues where a nurse completed the video-otoscopy recording ${ }^{14}$. Agreement in diagnosis was $81 \%$ and agreement on clinical management recommendations was $76 \% .^{14}$

Intra-rater concordance was substantial for asynchronous diagnosis using videootoscopy recording (otologist $\kappa=0.773$; GP $\kappa=0.737$ ), which corresponds to previous findings using asynchronous video-otoscopy images. ${ }^{11,12}$ The substantial inter-rater concordance between the diagnoses made in the present study suggest good agreement between diagnosis made by the otologist and the GP. Inter-rater concordance measured was equivalent to that reported in previous research between otolaryngologists. ${ }^{11,12}$ The inter- and intra-rater concordance in this study was also similar to the diagnostic concordance between onsite otomicroscopy and remote diagnosis. This suggests the variability of remote diagnosis, using video-otoscopy recordings, is similar to typical diagnostic variability that can be expected within and between clinicians. 
Video-otoscopy recordings could be used for asynchronous diagnosis to correctly identify ears without pathology more often in the current study (specificity $94.6 \%$ and $96.6 \%$ for otologist and GP respectively) than was previously reported for asynchronous diagnosis from video-otoscopy images $($ specificity $=89 \%) .{ }^{10}$ Negative predictive values for normal ears in the current study were equally high (negative predictive value 95.5 and 94.7 for otologist and GP respectively). Sensitivity values in the present study (sensitivity $77.6 \%$ and $72.4 \%$ for otologist and GP respectively) were lower than was previously reported for asynchronous diagnosis using video-otoscopy images however (sensitivity $80 \%$ and $85 \%$ for images acquired by an otolaryngologist and telehealth facilitator respectively). ${ }^{10}$

Video-otoscopy recordings may pose several advantages above video-otoscopy images for asynchronous diagnosis. Video-otoscopy recordings provide the possibility of pausing, rewinding and reviewing the recording several times, an opportunity rarely granted by a child. Compared to video-otoscopy images, video-otoscopy recordings appear to afford better depth perception as it offers several, dynamic angles of the tympanic membrane compared to a single still video-otoscopy image. ${ }^{10,11}$ Asynchronous diagnosis by the otologist compared to onsite assessment indicated slightly more CSOM ears. This may reflect the advantage of afforded by asynchronous assessments allowing several reviews with no time pressure as is the case when 
conducting onsite otomicroscopy with young children. Another advantage is that both the child and caregiver are able to see the ear canal and ear drum, providing good counselling and learning opportunities. In one case, a child co-operated willingly for video-otoscopy by the telehealth facilitator but not for the otomicroscopic examination. Video-otoscopy may be a less intimidating assessment for some children. Additionally a telehealth facilitator, who would likely originate from the community the clinic serves, and who speaks the child's home language, may also be perceived as less threatening than a doctor. The video-otoscope selected for the present study was portable and easy to operate with some training. In comparison with a surgical otomicroscope, the videootoscope is significantly less expensive. By increasing the telehealth facilitator's training and supervising period, the number of acceptable quality video-otoscopy recordings may increase, with fewer ears left undiagnosed after asynchronous assessment

\section{Conclusion}

A telehealth facilitator with limited training was capable of acquiring good quality video-otoscopy recordings in a paediatric sample for asynchronous diagnosis. Asynchronous video-otoscopy recordings have high intra- and inter-rater reliability for diagnoses made by an otologist and GP. Remote diagnosis was equivalent to inter- and 
intra-rater variability. However, asynchronous diagnosis could not be made for close to one in five paediatric video-otoscopy recordings due to residual cerumen in the ear canal or poor video-quality. Increasing the telehealth facilitator's training and supervising period and applying cerumen management strategies prior to videootoscopy recordings may reduce the number of ears left undiagnosed after asynchronous assessment.

\section{Acknowledgements}

The authors would like to thank Ms Violet Mugodo, the hearing telehealth clinic facilitator, Dr Jean Bassett, Executive Director of Witkoppen Health and Welfare Clinic, as well as the clinic staff and patients for their help and support during data collection for this research project. We are also grateful to Mr Headley Isserow at Tecmed, Midrand, South Africa, for providing the Leica otomicroscope used for on-site diagnoses, and to Dr Dirk Koekemoer, GeoAxon, for his continued support. Partial funding from the National Research Fund of South Africa is gratefully acknowledged.

\section{Declaration of Conflicting Interests}

The authors declare that there is no conflict of interest. 


\section{References}

1. Swanepoel DW, Clark JL, Koekemoer D, et al. Telehealth in audiology: The need and potential to reach underserved communities. Int J Audiol [Internet]. 2010 Mar [cited 2011 Jul 25];49(3):195-202. Available from:

http://www.ncbi.nlm.nih.gov/pubmed/20151929

2. Swanepoel DW, Hall JW III. A systematic review of telehealth applications in audiology. Telemed e-Health. 2010;16(2):181-201.

3. Monasta L, Ronfani L, Marchetti F, et al. Burden of disease caused by otitis media: Systematic review and global estimates. PLoS One [Internet]. 2012 Jan [cited 2012 Nov 17];7(4):1-12. Available from:

http://www.pubmedcentral.nih.gov/articlerender.fcgi?artid=3340347\&tool=pmce ntrez\&rendertype $=$ abstract

4. World Health Organization. Multi-country assessment of national capacity to provide hearing care [Internet]. Geneva, Switzerland: 2013. Available from: www.http://www.who.int/pbd/deafness/en

5. Acuin J. Chronic suppurative otitis media: Burden of Illness [Internet]. Geneva, Switzerland: 2004. Available from:

http://www.who.int/pbd/deafness/activities/hearing_care/otitis_media.pdf

6. World Health Organization/CIBA Foundation. Prevention of hearing impairment from chronic otitis media. London, UK: 1998.

7. Fagan JJ, Jacobs M. Survey of ENT services in Africa: Need for a comprehensive intervention. Glob Health Action [Internet]. 2009 Jan [cited 2011 Jul 31];2:1-8. Available from:

http://www.pubmedcentral.nih.gov/articlerender.fcgi?artid=2779942\&tool=pmce ntrez\&rendertype $=$ abstract

8. World Health Organization. Deafness and hearing impairment: Fact sheet [Internet]. 2013 [cited 2013 Jul 10];Available from: http://www.who.int/mediacentre/factsheets/fs300/en/

9. Lundberg T, Westman G, Hellström S, et al. Digital imaging and telemedicine as a tool for studying inflammatory conditions in the middle ear - Evaluation of 
image quality and agreement between examiners. Int J Pediatr Otorhinolaryngol [Internet]. 2008 Jan [cited 2010 Sep 1];72(1):73-79. Available from: http://www.ncbi.nlm.nih.gov/pubmed/17983668

10. Biagio L, Swanepoel DW, Adeyemo A, et al. Asynchronous video-otoscopy with a telehealth facilitator. Telemed e-Health. 2013;19(4):252-258.

11. Kokesh J, Ferguson AS, Patricoski C, et al. Digital images for postsurgical follow-up of tympanostomy tubes in remote Alaska. Otolaryngol - Head Neck Surg [Internet]. 2008 Jul [cited 2010 Sep 1];139(1):87-93. Available from: http://www.ncbi.nlm.nih.gov/pubmed/18585567

12. Patricoski C, Kokesh J, Ferguson AS, et al. A comparison of in-person examination and video otoscope imaging for tympanostomy tube follow-up. Telemed J e-Health [Internet]. 2003 Jan;9(4):331-344. Available from: http://www.ncbi.nlm.nih.gov/pubmed/14980090

13. Smith AC, Williams J, Agnew J, et al. Realtime telemedicine for paediatric otolaryngology pre-admission screening. J Telemed Telecare [Internet]. 2005 Jan;11(Suppl. 2):86-89. Available from: http://www.ncbi.nlm.nih.gov/pubmed/16375809

14. Smith AC, Perry C, Agnew J, et al. Accuracy of pre-recorded video images for the assessment of rural indigenous children with ear, nose and throat conditions. J Telemed Telecare [Internet]. 2006 Nov 1 [cited 2012 Apr 20];12(7):76-80. Available from: http://jtt.rsmjournals.com/content/12/suppl_3/76.abstract

15. Eikelboom RH, Mbao MN, Coates HL, et al. Validation of tele-otology to diagnose ear disease in children. Int J Pediatr Otorhinolaryngol [Internet]. 2005 Jun [cited 2010 Sep 1];69(6):739-744. Available from: http://www.ncbi.nlm.nih.gov/pubmed/15885325

16. Pedersen S, Hartviksen G, Haga D. Teleconsultation of patients with otorhinolaryngologic conditions. A telendoscopic pilot study. Arch Otolaryngol Head Neck Surg [Internet]. 1994 Feb [cited 2011 Apr 27];120(2):133-136. Available from: http://www.ncbi.nlm.nih.gov/pubmed/8297568

17. Smith AC, Dowthwaite S, Agnew J, et al. Concordance between real-time telemedicine assessments and face-to-face consultations in paediatric 
otolaryngology. Med J Aust [Internet]. 2008 Apr 21;188(8):457-60. Available from: http://www.ncbi.nlm.nih.gov/pubmed/18429711

18. Mbao MN, Eikelboom RH, Atlas MD, et al. Evaluation of video-otoscopes suitable for tele-otology. Telemed J e-Health. 2003;9(4):325-331.

19. American Speech-Language-Hearing Association. Audiologists providing clinical services via telepractice: Position statement [Internet]. 2005. Available from: www.asha.org/policy

20. Morris PS, Leach AJ. Acute and chronic otitis media. Pediatr Clin North Am [Internet]. 2009 Dec [cited 2012 Sep 24];56(6):1383-1399. Available from: http://www.ncbi.nlm.nih.gov/pubmed/19962027

21. Aronzon A, Ross AT, Kazahaya K, et al. Diagnosis of middle ear disease using tympanograms and digital imaging. Otolaryngol - Head Neck Surg [Internet]. 2004 Dec [cited 2012 Apr 20];131(6):917-920. Available from: http://www.ncbi.nlm.nih.gov/pubmed/15577789

22. Carruthers J. Dainfern and Diepsloot: Environmental justice and environmental history in Johannesburg, South Africa. Environ Justice [Internet]. 2008 Sep [cited 2013 Jan 31];1(3):121-126. Available from: http://www.liebertonline.com/doi/abs/10.1089/env.2008.0526

23. Bluestone CD, Gates GA, Klein JO, et al. Definitions, terminology, and classification of otitis media. Ann Otol Rhinol Laryngol. 2002;111:8-18.

24. Trochim WM. Research methods: Knowledge base [Internet]. Web Cent. Soc. Res. Methods. 2006 [cited 2010 Sep 11];Available from: http://www.socialresearchmethods.net/kb/index.php

25. Landis JR, Koch GG. The measurement of observer agreement for categorical data. Biometrics [Internet]. 1977;33(1):159-174. Available from: http://www.jstor.org/stable/2529310 